Article

\title{
Interferon-Induced Transmembrane Protein 1 (IFITM1) Promotes Distant Metastasis of Small Cell Lung Cancer
}

\author{
Shuichi Sakamoto ${ }^{1, * \mathbb{D}}$, Hiroyuki Inoue ${ }^{1}$, Yasuko Kohda ${ }^{1}$, Shun-ichi Ohba ${ }^{1}$, \\ Taketoshi Mizutani ${ }^{2}$ and Manabu Kawada 1,3 (D) \\ 1 Institute of Microbial Chemistry (BIKAKEN), 18-24 Miyamoto, Numazu 410-0301, Japan; \\ inoueh@bikaken.or.jp (H.I.); koday@bikaken.or.jp (Y.K.); ohbas@bikaken.or.jp (S.-i.O.); \\ kawadam@bikaken.or.jp (M.K.) \\ 2 Laboratory of Virology, Institute of Microbial Chemistry (BIKAKEN), 3-14-23 Kamiosaki, Shinagawa, \\ Tokyo 141-0021, Japan; miztanit@ims.u-tokyo.ac.jp \\ 3 Laboratory of Oncology, Institute of Microbial Chemistry (BIKAKEN), 3-14-23 Kamiosaki, Shinagawa, \\ Tokyo 141-0021, Japan \\ * Correspondence: sakamotos@bikaken.or.jp; Tel.: +81-55-924-0601
}

Received: 19 June 2020; Accepted: 10 July 2020; Published: 13 July 2020

\begin{abstract}
Small cell lung cancer (SCLC) is a severe malignancy associated with early and widespread metastasis. To study SCLC metastasis, we previously developed an orthotopic transplantation model using the human SCLC cell line DMS273. In the model, metastatic foci were found in distant tissues such as bone and the adrenal gland, similarly as observed in patients with SCLC. In this study, we evaluated the differentially expressed genes between orthotopic and metastatic tumors in the model. We isolated tumor cells from orthotopic and metastatic sites, and the tumor cell RNA was analyzed using DNA microarray analysis. We found that 19 genes in metastatic tumors were upregulated by more than 4 -fold compared with their expression in orthotopic tumors. One of these genes encodes a transmembrane protein, interferon (IFN)-induced transmembrane protein 1 (IFITM1), and immunohistochemical analysis confirmed the higher expression of the protein in metastatic sites than in orthotopic sites. IFITM1 was also detected in some SCLC cell lines and lung tumors from patients with SCLC. The overexpression of IFITM1 in DMS273 cells increased their metastatic formation in the orthotopic model and in an experimental metastasis model. Conversely, the silencing of IFITM1 suppressed metastatic formation by DMS273 cells. We also found that IFITM1 overexpression promoted the metastatic formation of NCI-H69 human SCLC cells. These results demonstrate that IFITM1 promotes distant metastasis in xenograft models of human SCLC.
\end{abstract}

Keywords: IFITM1; small cell lung cancer; metastasis; orthotopic transplantation model; experimental metastasis model

\section{Introduction}

Lung cancer is a leading cause of cancer-related death, and it is broadly classified into two types: non-small cell lung cancer (NSCLC) and small cell lung cancer (SCLC). SCLC comprises approximately $15 \%$ of all lung cancer cases, and it is characterized by rapid tumor growth, recurrence after chemotherapy, and early and widespread metastasis [1,2]. As a result of its aggressive nature, SCLC has a 5 -year survival rate of 5-10\% [3]. Although immune checkpoint inhibitors were recently approved for SCLC treatment, their efficacy remains limited to a small subset of patients [4]. Consequently, SCLC treatment has remained largely unchanged in recent decades, and the development of novel therapeutic strategies is urgently needed. Particularly, the inhibition of metastasis is important for 
improving treatment outcomes. Therefore, a better understanding of the mechanisms underlying SCLC metastasis is critical for the development of effective therapies.

Several studies have reported the factors promoting SCLC metastasis. Using xenograft mouse models generated using human SCLC cells, Dll4-Notch signaling and placental growth factor were both revealed to play critical roles in liver metastasis and brain metastasis, respectively [5,6]. Studies using genetically engineered mouse models of SCLC demonstrated that the transcription factors Pea3 and NFIB both promote metastasis [7-9]. However, compared with the findings in other cancers, the mechanisms underlying SCLC metastasis are largely unknown. Since surgery is rarely used to treat SCLC, it is difficult to obtain tumor samples, especially from metastatic sites, which represents a major obstacle in studying SCLC metastasis [1].

Previously, we developed an orthotopic transplantation model with a high incidence of metastasis using the human SCLC cell line DMS273 [10,11]. Based on the expression pattern of biochemical markers, SCLC cell lines can be subgrouped into two distinct classes: classic SCLC and variant SCLC. Under this classification, DMS273 cells can be classified as variant SCLC $[12,13]$. It was reported that variant SCLC cells had greater metastatic potential than classic SCLC cells in an experimental metastatic model, and this might be responsible for the high incidence of metastasis of our model $[10,12,14]$. In our model, it is relatively easy to obtain tumor samples from both orthotopic and metastatic tumors, which enables the evaluation of differentially expressed genes between orthotopic and metastatic tumors.

In this study, we performed a genome-wide mRNA microarray analysis of the orthotopic and metastatic tumors of our model, finding that 19 genes were upregulated by $>4$-fold in metastatic tumors compared with their levels in orthotopic tumors. One of the upregulated genes encodes interferon (IFN)-induced transmembrane protein 1 (IFITM1), also known as 9-27 or Leu13, which is a member of the IFN-inducible transmembrane protein family $[15,16]$. Transmembrane proteins play a pivotal role in mediating intercellular communication and signal transduction. The aberration of transmembrane proteins, such as overexpression and mutation, is frequently observed in cancer, and in many cases, this aberration is related to carcinogenesis and cancer progression, including metastasis [17]. IFITM1 is strongly induced by type I and II IFNs, and it has anti-viral effects against various viruses, including coronaviruses $[15,16,18-20]$. IFITM1 is also expressed in primordial germ cells and is required for their transition from the mesoderm into the endoderm through embryonic development in mice [21]. It was also demonstrated that IFITM1 was associated with cancer progression and metastasis in many cancers including glioma, colorectal cancer, head and neck cancer, and NSCLC [22-26]. However, its role in SCLC is unexplored. Therefore, we examined the role of IFITM1 in SCLC using a xenograft model mice.

\section{Results}

\subsection{IFITM1 Expression Was Higher in Metastatic Sites than in Orthotopic Sites in the Orthotopic SCLC Metastasis Model}

We previously developed a new orthotopic transplantation model using DMS273 cells [10]. In our model, distant metastatic foci were found in organs such as bone and the adrenal gland, as observed in patients with SCLC $[10,11]$. To explore the mechanism of SCLC metastasis using the orthotopic SCLC metastatic model, we sought to identify differentially expressed genes between orthotopic and metastatic tumors in the model. For this purpose, we isolated human tumor cells from three orthotopic lung tumors and four metastatic lesions (three bone metastases and one adrenal gland metastasis) using MACS ${ }^{\circledR}$ cell separation technology (Figure 1A,B). The total RNAs of the isolated tumor cells were analyzed using an Agilent SurePrint G3 Human GE $8 \times 60 \mathrm{~K}$ microarray that covered 60,000 transcripts to compare gene expression profiles between tumor cells from the orthotopic and metastatic sites. As a result, we identified 43 differentially expressed genes $(p<0.01$, fold change $>4)$ between the orthotopic and metastatic tumors (Figure 1C). Among these genes, 19 genes were upregulated by $>4$-fold in metastatic sites compared with their levels in orthotopic sites, and the most strongly overexpressed gene in the metastatic tumors was oncogenic long non-coding RNA H19, which was reported to 
promote cancer progression and metastasis in many cancers, including SCLC [27-30] (Table 1). Among the other overexpressed genes in the metastatic tumors, we focused on the IFITM1 gene, which encodes a small protein localized in the plasma membrane, because it was reported that this gene is involved in cancer progression and metastasis in several cancers; however, its roles in SCLC are unclear [22-25].

A

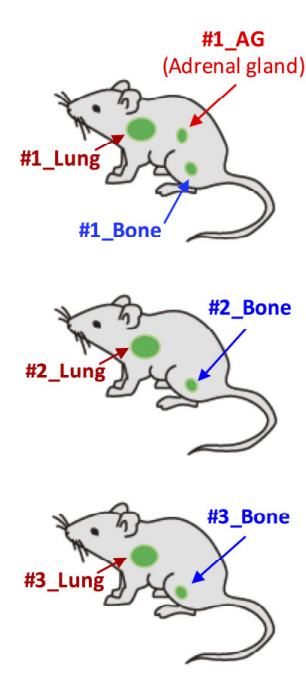

B

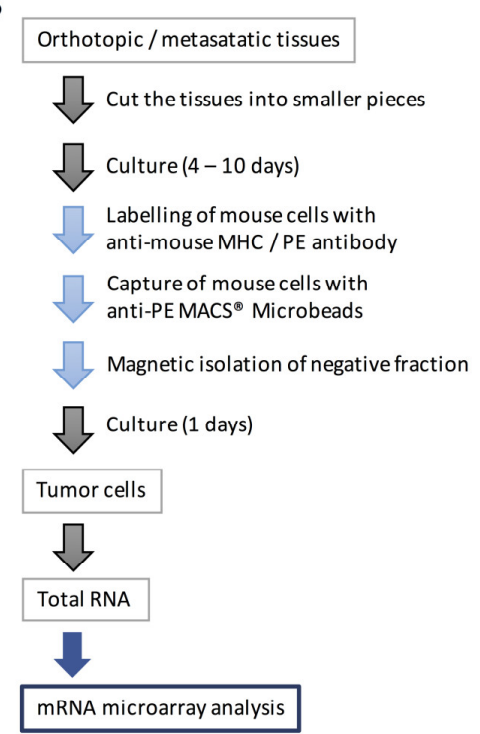

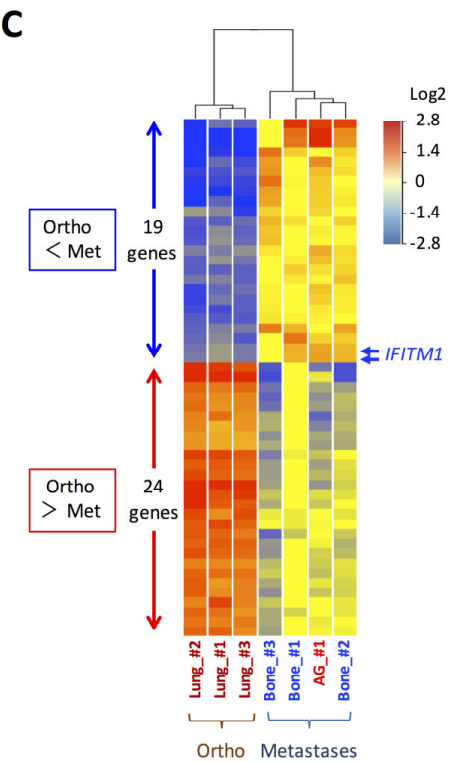

Figure 1. Gene expression analysis of tumor cells in orthotopic and metastatic sites of the orthotopic small cell lung cancer metastasis model. (A) Cells from the orthotopic and metastatic sites of the orthotopic metastasis model developed using DMS273 cells were subjected to DNA microarray analysis. (B) Schematic representation of the isolation of tumor cells from orthotopic and metastatic sites in the mice. (C) A clustering analysis of the 43 differentially expressed genes $(p<0.01$, fold change $>4)$ between the orthotopic and metastatic tumors.

Table 1. List of the 19 genes with higher expression at metastatic sites than at orthotopic sites $(p<0.01$, fold change $>4$ ) in the model. Comparison of the gene expression profiles of the three orthotopic tumors and four metastatic tumors revealed 21 probes with higher expression at metastatic sites than at orthotopic sites. The array includes one or more probes for each gene, and the 21 probes represent 19 genes.

\begin{tabular}{|c|c|c|c|c|}
\hline ProbeName & GeneSymbol & Description & Fold Change & $p$-Value \\
\hline A_24_P52697 & H19 & $\begin{array}{l}\text { Homo sapiens } \mathrm{H} 19 \text {, imprinted maternally } \\
\text { expressed transcript, long non-coding RNA }\end{array}$ & 26.05 & 0.001187 \\
\hline A_19_P00323082 & H19 & $\begin{array}{l}\text { Homo sapiens } \mathrm{H} 19 \text {, imprinted maternally } \\
\text { expressed transcript, long non-coding RNA }\end{array}$ & 25.55 & 0.001265 \\
\hline A_23_P13753 & NFE2 & $\begin{array}{l}\text { Homo sapiens nuclear factor, erythroid } 2 \\
\text { (NFE2), transcript variant } 1\end{array}$ & 16.41 & 0.003368 \\
\hline A_23_P104188 & ELF3 & $\begin{array}{l}\text { Homo sapiens E74-like factor 3, transcript } \\
\text { variant } 1\end{array}$ & 15.41 & 0.001043 \\
\hline A_23_P17190 & KLHL41 & Homo sapiens kelch-like family member 41 & 9.79 & 0.000221 \\
\hline A_33_P3348061 & CABP7 & Homo sapiens calcium binding protein 7 & 8.56 & 0.000461 \\
\hline A_33_P3209229 & RAB26 & $\begin{array}{l}\text { Homo sapiens RAB26, member RAS } \\
\text { oncogene family }\end{array}$ & 8.52 & 0.001577 \\
\hline A_33_P3244808 & BEST4 & Homo sapiens bestrophin 4 & 8.45 & 0.001459 \\
\hline A_32_P131143 & CECR5-AS1 & $\begin{array}{l}\text { Homo sapiens CECR5 antisense RNA 1, } \\
\text { transcript variant } 2 \text {, long non-coding RNA }\end{array}$ & 7.55 & 0.000385 \\
\hline A_23_P302005 & STON1 & Homo sapiens stonin 1 , transcript variant 2 & 6.60 & 0.001385 \\
\hline A_32_P356316 & HLA-DOA & $\begin{array}{l}\text { Homo sapiens major histocompatibility } \\
\text { complex, class II, DO alpha }\end{array}$ & 6.04 & 0.002569 \\
\hline
\end{tabular}


Table 1. Cont.

\begin{tabular}{cclcc}
\hline ProbeName & GeneSymbol & \multicolumn{1}{c}{ Description } & Fold Change & $p$-Value \\
\hline A_23_P130753 & DBP & $\begin{array}{l}\text { Homo sapiens D site of albumin promoter } \\
\text { (albumin D-box) binding protein }\end{array}$ & 5.59 & 0.000157 \\
A_24_P59667 & JAK3 & $\begin{array}{l}\text { Homo sapiens Janus kinase 3 } \\
\text { Homo sapiens RAD52 motif containing 1, } \\
\text { transcript variant 2 }\end{array}$ & 5.43 & 0.000024 \\
A_23_P141447 & RDM1 & 5.23 & 0.000003 \\
A_33_P3423941 & IFITM1 & $\begin{array}{l}\text { Homo sapiens interferon induced } \\
\text { transmembrane protein 1 }\end{array}$ & 5.18 & 0.000986 \\
A_23_P78108 & ALDOC & $\begin{array}{l}\text { Homo sapiens aldolase C, } \\
\text { fructose-bisphosphate }\end{array}$ & 5.09 & 0.000254 \\
A_24_P521994 & KLHL24 & $\begin{array}{l}\text { Homo sapiens kelch-like family member 24 } \\
\text { Homo sapiens interferon induced }\end{array}$ & 5.02 & 0.000151 \\
A_23_P72737 & IFITM1 & $\begin{array}{l}\text { transmembrane protein 1 } \\
\text { Homo sapiens zinc finger CCCH-type } \\
\text { containing 6 }\end{array}$ & 4.98 & 0.000986 \\
A_24_P826348 & ZC3H6 & 4.20 & 0.000294 \\
A_23_P382775 & BBC3 & $\begin{array}{l}\text { Homo sapiens BCL2 binding component 3, } \\
\text { transcript variant 4 }\end{array}$ & 4.19 & 0.000599 \\
A_23_P216023 & ANGPT1 & $\begin{array}{l}\text { Homo sapiens angiopoietin 1, transcript } \\
\text { variant 1 }\end{array}$ & 4.08 & 0.002805 \\
\hline
\end{tabular}

We examined IFITM1 mRNA expression levels in isolated tumor cells subjected to the DNA microarray analysis by real-time RT-PCR and confirmed that its expression was higher in metastatic tumors than in orthotopic tumors (Figure 2A). Subsequently, we performed IFITM1 immunostaining in orthotopic tumors and their corresponding metastatic tumors from mice and found that IFITM1 protein levels were higher in metastatic tumors than in orthotopic tumors (Figure 2B,C). Thus, IFITM1 expression is elevated in metastatic tumors compared with that in orthotopic tumors in our orthotopic SCLC metastasis model.

A

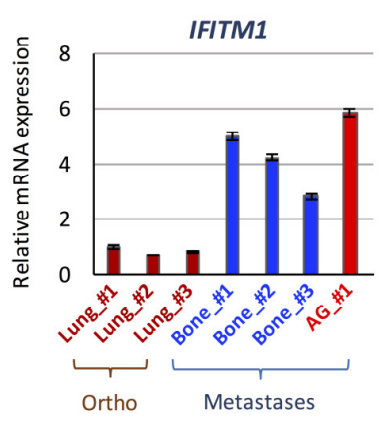

B

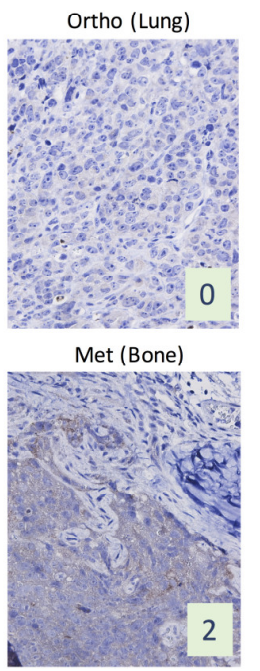

Met (Adrenal gland)

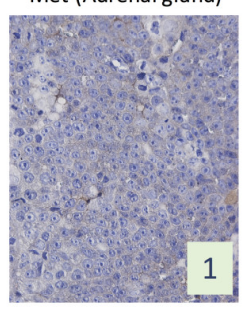

Met (Adrenal gland)

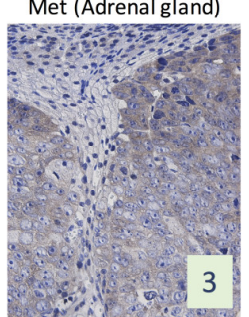

C

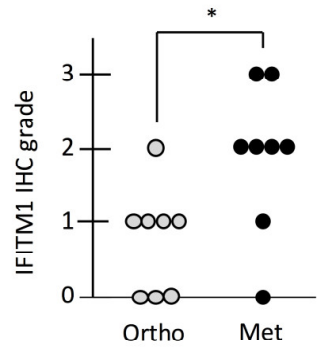

Figure 2. Expression of interferon (IFN)-induced transmembrane protein 1 (IFITM1) in the orthotopic small cell lung cancer (SCLC) metastasis model. (A) Quantitative analysis of IFITM1 mRNA in tumor cells subjected to the DNA microarray analysis. In total, $1 \mu \mathrm{g}$ of total RNA from the tumor cells was subjected to real-time RT-PCR. Data are expressed as the mean \pm SD of triplicate experiments. (B) Representative immunohistochemistry images for IFITM1. Images of IFITM1 staining of an orthotopic tumor and three distant metastases of the orthotopic models using DMS273 cells are shown. The images were taken at $\times 400$ magnification. Numbers represent the immunohistochemical scores. The estimated visual intensity of IFITM1 staining was graded on an arbitrary 4-point scale as follows: negative, 0; weakly positive, 1; positive, 2; and strongly positive, 3. (C) Summary of immunohistochemical analysis of IFITM1 expression in SCLC tumors. The immunohistochemical scores of the orthotopic tumors of eight mice and their corresponding distant metastases were calculated. * $p<0.05$, Mann-Whitney U-test. 


\subsection{IFITM1 Expression in Human SCLC Cell Lines and Lung Tumor Tissues from Patients with SCLC}

We next investigated IFITM1 expression in human SCLC cell lines and lung tumor tissues from patients with SCLC. Western blot analysis of four human SCLC cell lines revealed that IFITM1 protein was expressed in DMS273 and DMS114 cells, but it was not detectable in NCI-H69 and DMS53 cells (Figure 3A). Since human IFITM1 is strongly induced by IFNs [31,32], we examined the effect of IFNs on IFITM1 expression in DMS273-GFP cells and H69ZN cells (a ZsGreen-labeled subline of NCI-H69 cells). Western blot analysis revealed that the IFITM1 protein was upregulated by treatment with IFN $\alpha$, IFN $\beta$, or IFN $\gamma$ in DMS273-GFP cells (Figure 3B). Conversely, IFITM1 protein expression was upregulated by treatment with IFN $\alpha$ or IFN $\beta$ in H69ZN cells (Figure 3B). Next, we investigated IFITM1 protein levels in primary lung tumor tissues from patients with SCLC using a commercially available cancer tissue array (CTA). As shown in Figure 3C,D, immunostaining on the CTA demonstrated that IFITM1 was expressed in 30.6\% (26/85) of primary SCLC tumors but not in any normal lung tissue samples $(0 / 8)$.

A

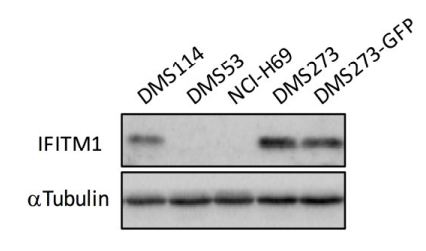

B

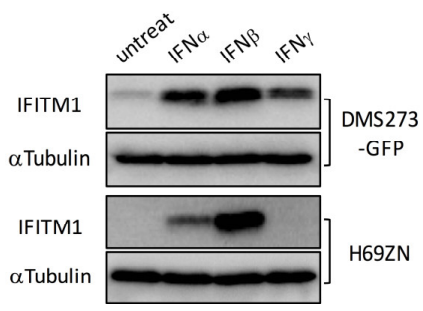

C
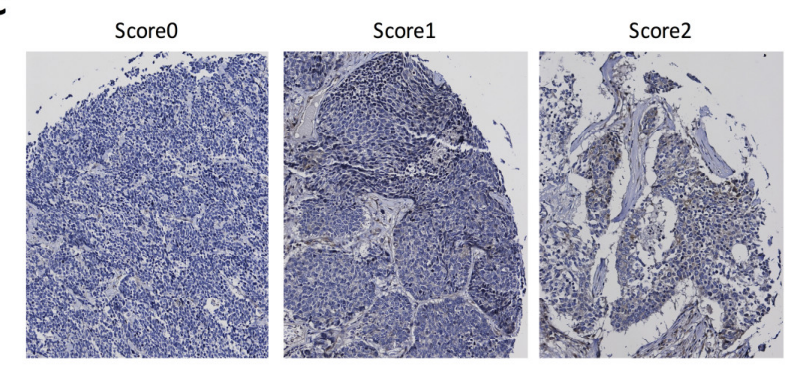

D

$\left.\begin{array}{cccccc}\hline & \text { Total No. } & \text { Score 0 } & \text { Score 1 } & \text { Score 2 } & \text { Positive (\%) } \\ \hline \begin{array}{c}\text { SCLC } \\ \text { lung tumor }\end{array} & 85 & 59 & 21 & 5 & 30.6 \\ \text { Normal lung } & 8 & 8 & 0 & 0 & 0\end{array}\right]$ n.s.

Figure 3. IFITM1 expression in human SCLC cell lines and lung tumor tissues from patients with SCLC. (A) Western blotting for IFITM1 in human SCLC cell lines. Whole-cell lysates (20 $\mu \mathrm{g})$ were separated by $15 \%$ SDS-PAGE, and membranes were blotted with anti-IFITM1 (top panel) and anti- $\alpha$-tubulin antibodies (bottom panel, loading control). (B) Western blotting for IFITM1 in IFN-treated DMS273-GFP cells and H69ZN cells (a ZsGreen-labeled subline of NCI-H69 cells). Whole-cell lysates (20 $\mu \mathrm{g}$ ) were separated by $15 \%$ SDS-PAGE. (C) Representative immunohistochemistry images of IFITM1 staining in lung tumor tissues from patients with SCLC on cancer tissue arrays. The images were taken at $\times 200$ magnification. (D) Summary of the cancer tissue array analysis. The estimated visual intensity of IFITM1 immunostaining was graded on an arbitrary 3-point scale as follows: negative, 0; positive, 1; and strongly positive, 2. n.s.; not significant, Fisher's exact test.

\subsection{IFITM1 Overexpression Promotes Metastatic Formation by DMS273 Cells in Nude Mice}

To assess the role of IFITM1 in metastatic formation in the orthotopic SCLC metastasis model, we first established an IFITM1-overexpressing subline of DMS273-GFP cells [10] using a lentivirus vector (Figure 4A). The IFITM1-overexpressing subline exhibited similar rates of in vitro growth, anchorage-independent growth, migration, and invasion as the control vector-transfected subline (Figure 4B and Figure S1a-c). In the orthotopic SCLC metastasis model, the IFITM1-overexpressing subline exhibited a significantly higher rate of metastasis than the vector control subline $(95 \%$ versus $65 \%, p<0.05$, Figure $4 C, D)$. Conversely, the orthotopic lung tumor growth of the IFITM1-overexpressing subline was not different from that of the control vector subline (Figure $4 \mathrm{E}$ ). We did not observe a significant difference of metastatic tropism between these sublines in this model (Figure S1d). We also examined the effect of IFITM1 overexpression on distant metastasis using an experimental 
metastasis model involving tail vein injection in nude mice. The IFITM1-overexpressing subline formed significantly more nodules than the control vector subline $(p<0.01$, Figure $4 \mathrm{~F}, \mathrm{G})$. We further demonstrated that IFITM1 overexpression did not affect in vivo subcutaneous tumor growth in nude mice (Figure $4 \mathrm{H}$ ). Thus, IFITM1 overexpression significantly enhanced distant metastatic formation but did not affect the in vivo tumor growth of DMS273-GFP cells.

A

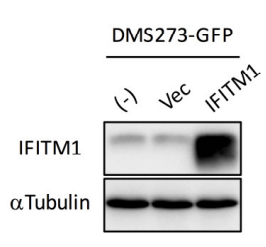

\section{E}

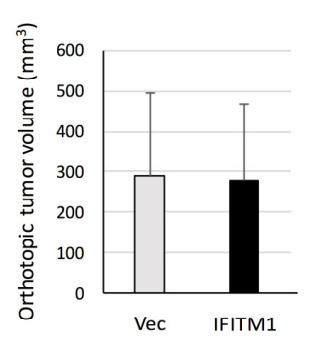

B

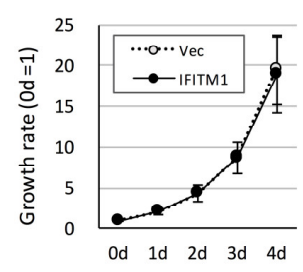

$\mathbf{F}$

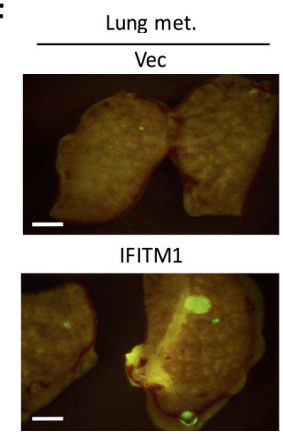

C

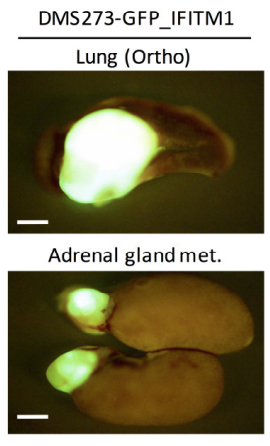

G

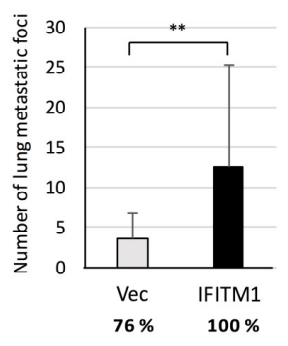

D

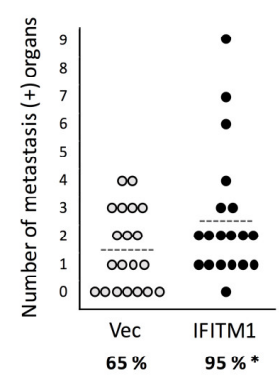

H

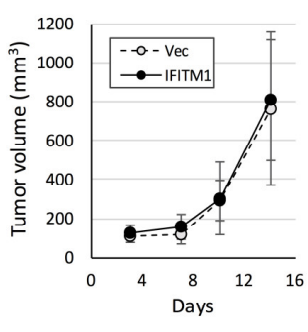

Figure 4. Effect of IFITM1 overexpression on the metastatic formation of DMS273-GFP cells in nude mice. (A) Western blotting for IFITM1 in IFITM1-overexpressing DMS273-GFP cells. Whole-cell lysates $(20 \mu \mathrm{g})$ were separated by $15 \%$ SDS-PAGE, and membranes were blotted with anti-IFITM1 (top panel) and anti- $\alpha$-tubulin antibodies (bottom panel, loading control). (B) In vitro growth rate of IFITM1-overexpressing cells as determined using the 3-(4,5-dimethylthiazol-2-yl)- 2,5 diphenyltetrazolium bromide (MTT) assay. The growth rate was calculated as the ratio of the absorbance of cultured cells to that of cells on day 0 . Data are expressed as the mean \pm SD of three independent experiments performed in triplicate. (C-E) Orthotopic tumor growth and metastatic formation in the orthotopic model using DMS273-GFP vector control cells $(n=19)$ or IFITM1-overexpressing cells $(n=20)$. In total, $2.5 \times 10^{5}$ cells were transplanted into the left lung of each nude mouse. One hundred micrograms of anti-asialo $\mathrm{GM}_{1}$ were injected into mice intravenously before and after tumor inoculation (a total of four injections). Mice were sacrificed when they became moribund, and orthotopic and metastatic tumors were assessed. (C) Representative fluorescence images of the orthotopic tumors and adrenal gland metastases of mice transplanted with IFITM1-overexpressing cells. Bar, $2 \mathrm{~mm}$. (D) Distant metastatic tumor formation. The dotted lines represent the means of the number of metastasis-positive organs per mouse. Percentages show the incidence of distant metastasis. ${ }^{*} p<0.05$, Fisher's exact test. (E) Orthotopic tumor formation. Results are expressed as the mean + SD. $(F, G)$ Metastatic colony formation in lungs in experimental metastasis models generated using DMS273-GFP vector control $(n=17)$ or IFITM1-overexpressing cells $(n=14)$. In total, $1 \times 10^{6}$ cells were injected into the tail vein of each nude mouse. At 7 weeks post-inoculation, lungs were dissected, and metastatic foci were counted. (F) Representative fluorescence images of the lung metastases of both groups. Bar, $2 \mathrm{~mm}$. (G) Percentages show the incidence of distant metastasis. ${ }^{* *} p<0.01$, Mann-Whitney U-test. (H) Subcutaneous tumor growth of DMS273-GFP vector control and IFITM1-overexpressing cells. Cells $\left(1 \times 10^{6}\right)$ were inoculated subcutaneously in nude mice $(n=6)$. The tumor volumes are shown as the mean $\pm \mathrm{SD}$. 


\subsection{Silencing of IFITM1 Suppresses Metastatic Formation by DMS273 Cells in Nude Mice}

Next, we generated a stable IFITM1-silenced subline of DMS273-GFP cells using lentivirus vector-based short hairpin RNA (shRNA) and examined the effect on the metastatic activity of the cells (Figure 5). Real-time RT-PCR analysis demonstrated that shRNA transfection effectively reduced IFITM1 mRNA expression in DMS273-GFP cells (Figure 5A). Western blot analysis confirmed that shRNA transfection effectively reduced IFITM1 protein levels in both IFN $\beta$ treated and untreated groups (Figure 5B). The IFITM1-silenced subline of DMS273-GFP cells exhibited similar rates of in vitro growth, anchorage-independent growth, migration, and invasion as the control shRNA (shLacZ)-transfected subline (Figure 5C and Figure S2a-c). In the orthotopic SCLC metastasis model, the IFITM1-silenced subline exhibited a significantly lower number of metastasis-positive organs than control shRNA cells $(p<0.05$, Figure 5D). Conversely, orthotopic tumor growth was not affected by IFITM1 silencing (Figure 5E). In an experimental metastasis model, the IFITM1-silenced subline also displayed a significantly lower number of lung metastatic foci $(p<0.05)$ and significantly lower rate of distant metastases $(p<0.05$, Figure 5F). Thus, the silencing of IFITM1 suppresses the metastatic ability of DMS273-GFP cells. Taken together, our results clearly demonstrate that IFITM1 enhances distant metastasis formation in the xenograft models of human DMS273 cells.
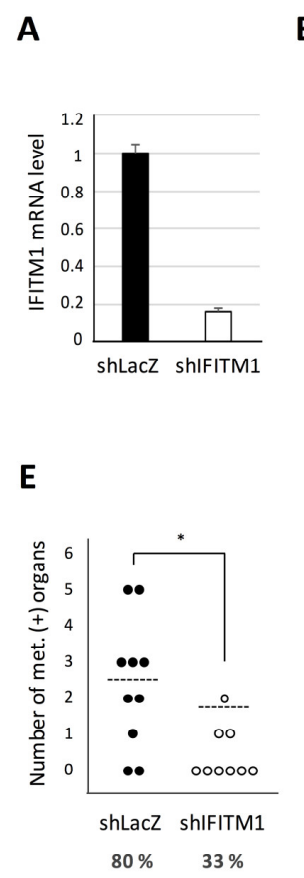

B

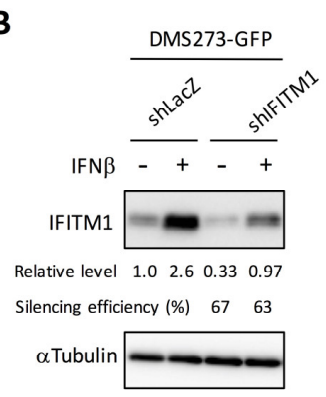

$\mathbf{F}$

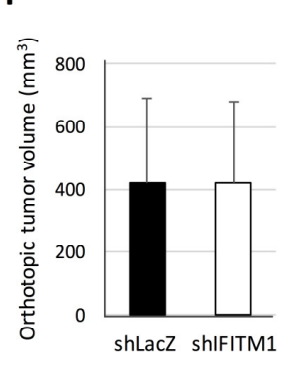

C

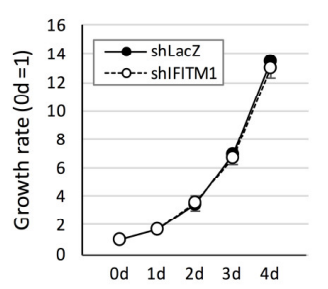

G

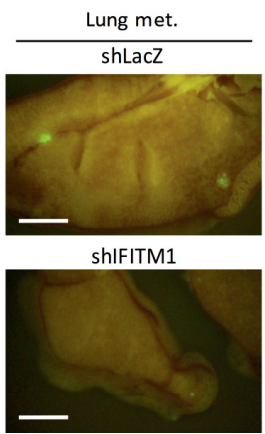

D

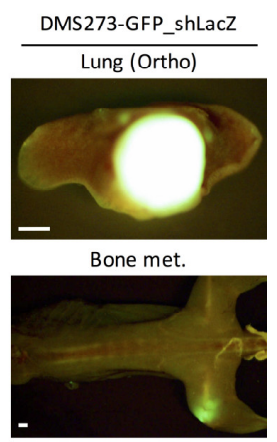

H

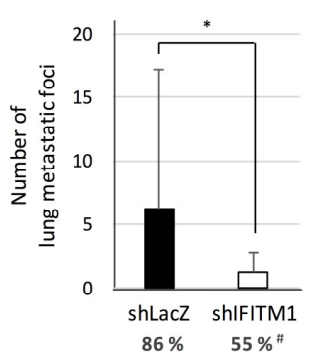

Figure 5. Effect of IFITM1 silencing on the metastatic formation of DMS273-GFP cells in nude mice. (A) Quantitative analysis of IFITM1 mRNA in silenced cells. One microgram of total RNA extracted from the cells was subjected to real-time RT-PCR. Data are expressed as the mean $+\mathrm{SD}$ of a triplicate experiment. (B) Western blotting for IFITM1. The cells were treated with $10 \mathrm{ng} / \mathrm{mL}$ interferon $\beta$ (IFN $\beta$ ) for $24 \mathrm{~h}$ and then lysed. Whole-cell lysates (20 $\mu \mathrm{g}$ ) were separated by 15\% SDS-PAGE, and membranes were blotted with anti-IFITM1 (top panel) and anti- $\alpha$-tubulin antibodies (bottom panel, loading control). Relative IFITM1 expression was calculated from the signal intensity and normalized to $\alpha$-tubulin levels. Silencing efficiency was calculated as the percentage of the relative expression level in shIFITM1 cells to that in shLacZ cells. (C) The in vitro growth rate of the silenced cells was determined using the MTT assay. The growth rate was calculated as the ratio of the absorbance of cultured cells to that of cells on day 0 . Data are expressed as the mean $\pm \mathrm{SD}$ of three independent experiments performed in triplicate. (D-F) Orthotopic tumor growth and metastatic formation in mice with orthotopic tumors generated 
using DMS273-GFP control shRNA (shLacZ) $(n=10)$ or IFITM1-silenced (shIFITM1) cells $(n=9)$. In total, $2.5 \times 10^{5}$ cells were transplanted into the left lung of each nude mouse. Mice were sacrificed when they became moribund, and orthotopic and metastatic tumors were assessed. (D) Representative fluorescence images of the orthotopic tumor and bone metastases of mice transplanted with control shRNA (shLacZ) cells. Bar, $2 \mathrm{~mm}$. (E) Distant metastatic tumor formation. The dotted lines represent the means of the number of metastasis-positive organs per mouse. Percentages show the incidence of distant metastasis in each group. ${ }^{*} p<0.05$, Mann-Whitney U-test. (F) Orthotopic tumor formation. Results are expressed as the mean + SD. (G-H) Metastatic colony formation in the lungs of experimental metastasis models generated using DMS273-GFP control shRNA (shLacZ) $(n=22)$ or IFITM1-silenced (shIFITM1) cells $(\mathrm{n}=20)$. In total, $1 \times 10^{6}$ cells were injected into the tail vein of each nude mouse. At 7-8 weeks post-inoculation, lungs were dissected, and metastatic foci were counted. (G) Representative fluorescence images of the lung metastases of both groups. Bar, $2 \mathrm{~mm}$. (H) Percentages show the incidence of distant metastasis. ${ }^{*} p<0.05$, Mann-Whitney U-test. \# $p<0.05$, Fisher's exact test.

\subsection{Overexpression of IFITM1 Promotes Metastatic Formation by NCI-H69 Cells in Nude Mice}

Next, we investigated the role of IFITM1 in the metastatic formation of another human SCLC cell line, namely NCI-H69, which was classified as a classic SCLC cell line [12]. We established an IFITM1-overexpressing subline of H69ZN cells (Figure 6A), and the cells were inoculated into nude mice via tail vein injection. In this model, distant metastatic foci were found in many tissues such as the lungs, brain, and adrenal glands (Figure 6B). As shown in Figure 6C, the IFITM1-overexpressing H69ZN cells formed significantly more metastatic foci than control vector-transfected cells $(p<0.01)$ in the model. Interestingly, the IFITM1-overexpressing subline formed significantly more distant metastases in the lungs $(p<0.01)$ and ovaries $(p<0.01)$ than did the control vector control subline (Figure 6D). Thus, IFITM1 overexpression promotes metastatic formation by NCI-H69 cells in nude mice.

A

B H69ZN-IFITM1
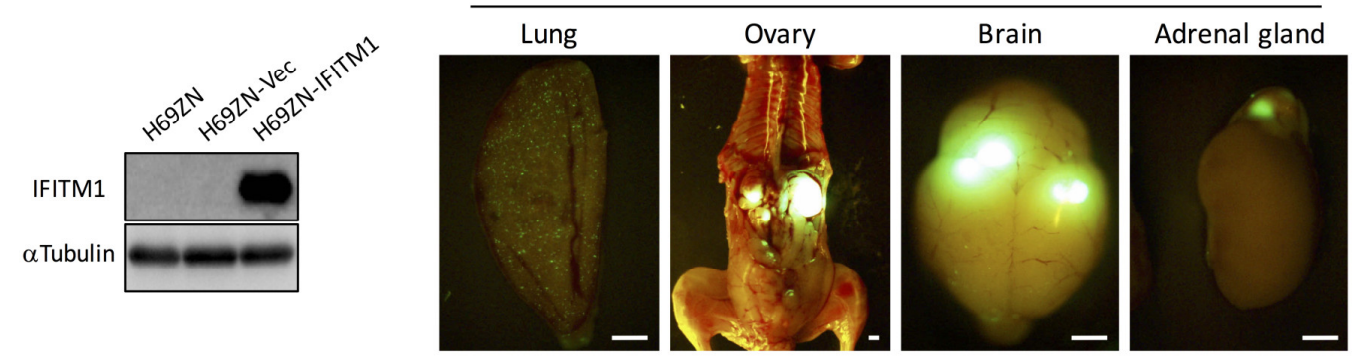

C

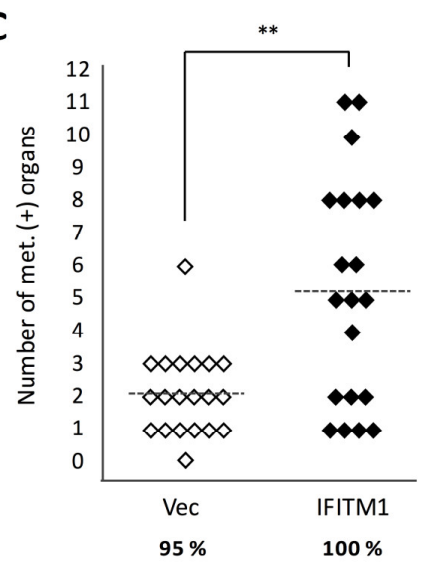

D

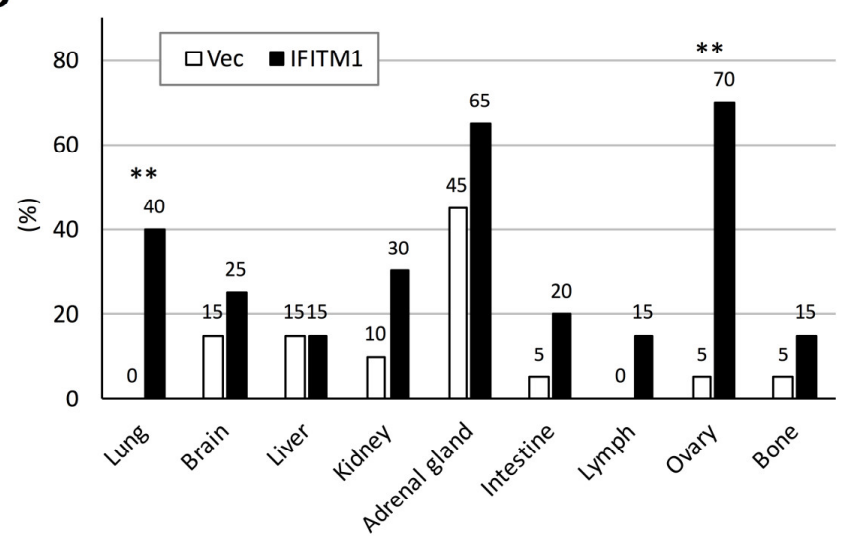

Figure 6. Effect of IFITM1 overexpression on the metastatic formation of NCI-H69 cells in nude mice. (A) Western blotting of IFITM1 expression in H69ZN cells. Whole-cell lysates (20 $\mu \mathrm{g})$ were separated 
by $15 \%$ SDS-PAGE, and membranes were blotted with anti-IFITM1 (top panel) and anti- $\alpha$-tubulin antibodies (bottom panel, loading control). (B) Representative fluorescence images of metastases in the lungs and ovaries of mice inoculated with IFITM1-overexpressing cells. Bar, $2 \mathrm{~mm}$. (C) Metastatic tumor formation in the experimental metastatic models generated using H69ZN vector control $(n=20)$ and IFITM1-overexpressing cells $(n=20)$. In total, $4 \times 10^{6}$ cells were injected into the tail vein of each nude mouse. At 6 weeks post-inoculation, mice were sacrificed, and metastatic tumors were assessed. Percentages show the incidence of distant metastasis of each group. ${ }^{* *} p<0.01$, Mann-Whitney U-test. (D) Organ distribution of metastases in the experimental metastatic models generated using H69ZN vector control and IFITM1-overexpressing cells. Data represent the percentage of metastasis-positive mice. ${ }^{* *} p<0.01$, Fisher's exact test.

\section{Discussion}

In this study, we compared the gene expression profiles of orthotopic and metastatic tumors in an orthotopic metastasis model using DMS273 cells and identified IFITM1 as a highly upregulated protein in metastatic sites (Figures 1 and 2). IFITM1 protein expression was also detected in some SCLC cell lines and lung tumors from patients with SCLC, and the protein was induced by IFN treatment in SCLC cell lines (Figure 3). Overexpression and silencing experiments demonstrated that IFITM1 enhanced metastatic formation without altering the in vivo tumor growth of DMS273 cells (Figures 4 and 5). Furthermore, we also found that IFITM1 overexpression promotes metastatic formation by NCI-H69 cells (Figure 6). These results demonstrate that IFITM1 promotes distant metastasis in xenograft models of human SCLC cell lines and suggest that IFITM1 enhances metastatic formation in patients with SCLC. Although several reports found that IFITM1 promoted tumor metastasis in various cancers including NSCLC $[25,26]$, this is the first report to our knowledge demonstrating that IFITM1 plays an important role in SCLC metastasis.

Since SCLC is not amenable to surgery, it is difficult to obtain primary and metastatic tumors from patients with SCLC, which has hindered the study of SCLC metastasis. Genetically engineered mouse models of SCLC represent one good solution to this obstacle. Denny et al. performed a molecular analysis of genome-wide chromatin accessibility in primary and metastatic tumor cells isolated from a genetically engineered SCLC model mice [8]. They found that metastases in the SCLC model mice have profound chromatin changes compared with the findings in primary tumors, and these changes were mediated by the transcription factor NFIB, indicating that NFIB promotes the metastasis of SCLC cells. Semanova et al. also demonstrated the pivotal role of NFIB in SCLC progression and metastasis using another genetically engineered mouse model [9]. In this study, we used an orthotopic metastasis model of SCLC and identified IFITM1 as a promoting factor for metastasis. Although Denny et al. reported that NFIB expression tended to be higher in distant metastases [8], we did not observe a significant increase in NFIB expression in metastatic tumor cells in our microarray analysis (data not shown). This may reflect the difference of the study models, which enabled us to discover another metastasis-promoting factor. Thus, our orthotopic xenograft model provides an alternative model for the study of SCLC metastasis in addition to genetically engineered mouse models.

Whereas IFITM1 expression was only detected in some SCLC cell lines and lung tumors from patients with SCLC (Figure 3), the tumor cells isolated from metastatic sites highly expressed IFITM1 compared with its levels in tumor cells from orthotopic sites in our SCLC metastasis model (Figure 2). Moreover, immunohistochemical analysis confirmed the higher IFITM1 protein expression in metastatic sites than in orthotopic sites (Figure 2). Consistent with our observation, a previous study in colorectal cancer demonstrated that IFITM1 expression was significantly higher in liver metastatic regions than in normal and tumor regions of patient-derived samples [24]. Additionally, it has been demonstrated that IFITM1 protein expression was observed at the invasive front of early invasive head and neck squamous cell carcinoma (HNSCC) lesions, and higher expression of the protein was detected in invasive HNSCC [22]. These observations support the idea that IFITM1 plays an important role in metastasis in several cancers. Notably, Sari et al. reported that increased IFITM1 expression is associated with poor prognosis in colorectal cancer [24], and other groups obtained similar findings 
in colorectal cancer and lung adenocarcinoma [23,25]. These studies suggest that IFITM1 is a good candidate for a poor prognostic marker and therapeutic target for those cancers. A similar relationship between IFITM1 expression and poor prognosis might exist in SCLC, but further analysis is needed to address the possibility.

The human IFITM family comprises five members, namely IFITM1, IFITM2, IFITM3, IFITM5, and IFITM10 [33]. Among these members, the role of IFITM3 in cancer is becoming apparent similarly as IFITM1. In a study in colorectal cancer, IFITM3 was expressed at higher levels in tumors, especially in nodal metastases, than in normal colon tissue, and the silencing of IFITM3 suppressed tumor growth and metastasis in a xenograft model of HCT116 colon cancer cells [34]. IFITM3 also promoted metastasis in xenograft models of hepatocellular carcinoma and prostate cancer $[35,36]$. Thus, IFITM3 is also an important factor for tumor metastasis in several cancers, but we did not observe any upregulation of IFITM3 in metastatic sites in the orthotopic SCLC metastasis model (data not shown). There are some differences in the subcellular localization of IFITM1 and IFITM3 [37], and mouse Ifitm1 and Ifitm 3 play distinct roles in primordial germ cell transition and homing [21]. Therefore, these two IFITM proteins might also have distinct roles in tumor metastasis.

Although our results demonstrated that IFITM1 promotes metastatic formation by two human SCLC cell lines in nude mice, the mechanism remains to be unveiled. It was reported that IFITM1 is involved in the invasion and/or metastasis of various cancers including glioma, colorectal cancer, ovarian cancer, head and neck cancer, and NSCLC [22-25,38,39]. In many of these cancers, it was demonstrated that IFITM1 is also involved in tumor growth in vitro and/or in vivo. By contrast, we observed that IFITM1 promoted tumor metastasis without any apparent effects on tumor growth, migration, and invasion in DMS273 cells under our experimental conditions (Figures 4 and 5, Figures S1 and S2). These results imply that the mechanism underlying the promotion of metastasis by IFITM1 in SCLC is somewhat different from those in other cancers. One possible mechanism is enhancing adhesion to cells and/or extracellular matrices. It has been reported that IFITM1 generally localizes to the plasma membrane [37] and participates in homotypic adhesion signal transduction in lymphocytes [40]. In addition, IFITM1 has been revealed to localize to tight junctions in hepatocytes and promote the redistribution of the membranous protein CD81 [41]. Therefore, it is possible that IFITM1 alters the affinity of SCLC cells for other cells and/or extracellular matrices, thereby enhancing SCLC metastasis. Another possible mechanism is escape from immune surveillance. Yang et al. reported that IFITM1 overexpression rendered gastric tumor cells more resistant to natural killer cells, which play a major role in the host rejection of tumor cells [42]. The increased expression of IFITM1 may also render SCLC cells resistant to natural killer cells, and the cells may in turn be able to escape from tumor immune surveillance.

We confirmed that IFITM1 was strongly induced by IFNs in SCLC cell lines (Figure 3B). The constitutive expression of IFNs and activation of their signaling pathways have critical roles in host responses to malignant cells in the tumor microenvironment [43]. Therefore, it is possible that IFITM1 expression in SCLC cells was induced by IFNs in the tumor microenvironment. The strong induction of IFITM1 may occur in a small portion of tumor cells in the primary tumor tissues because we detected relatively weak IFITM1 expression in the orthotopic tumors in our model and the primary tumors of patients with SCLC (Figures 2 and 3). The strongly induced IFITM1 should amplify the metastatic potential of SCLC cells, possibly by enhancing the adhesion ability of SCLC cells and/or their resistance to natural killer cells, and these tumor cells would ultimately form distant metastases.

In summary, we identified IFITM1 as a metastasis-promoting factor for SCLC by analyzing differentially expressed genes between orthotopic and metastatic tumors in an SCLC xenograft model. Further studies are required to clarify the mechanism by which IFITM1 promotes metastasis in SCLC. These further studies would provide useful clues to understand SCLC metastasis and develop new therapies for SCLC. 


\section{Materials and Methods}

\subsection{Reagents}

The pZsGreen-C1 vector was obtained from Clontech/Takara Bio (Shiga, Japan). IFN $\alpha 2 a$ was purchased from Proteintech (Rosemont, IL, USA). IFN $\beta$ and IFN $\gamma$ were purchased from PeproTech (Rocky Hill, NJ, USA). Anti-asialo GM $_{1}$ serum was obtained from FUJIFILM Wako Pure Chemical Corporation (Osaka, Japan). G418 was purchased from Thermo Fisher Scientific (Waltham, MA, USA).

\subsection{Cell Lines}

DMS273 cells and their GFP-labeled sublines [10] were maintained in DMEM (Nissui Pharmaceutical, Tokyo, Japan) containing 10\% FBS (PAN Biotech, Aidenbach, Germany). NCI-H69, DMS53, and DMS114 human SCLC cells were maintained in RPMI 1640 medium containing 10\% FBS. For ZsGreen labeling in NCI-H69 cells, the pZsGreen-C1 plasmid was transfected into cells using the Neon Transfection System (Thermo Fisher Scientific). ZsGreen-labeled cells were selected using G418-containing culture medium and named H69ZN cells.

\subsection{In Vitro Growth Assay}

For the in vitro cell growth assay, cells were seeded in normal 96-well plates (SUMILON, Tokyo, Japan) at a density of $1 \times 10^{3}$ cells in $100 \mu \mathrm{L}$ of DMEM containing $10 \%$ FBS. For anchorage-independent growth, cells were seeded in a low attachment plate (PrimeSurface 96-well U plates, SUMILON) and a normal 96-well plate at a density of $1 \times 10^{3}$ cells in $100 \mu \mathrm{L}$ of DMEM containing $2 \%$ FBS. After culture for the indicated times, cell growth was determined with using the MTT assay.

\subsection{Wound-Healing Assay}

Confluent cells in 24-well plates were carefully scratched with the tip of a 200- $\mu \mathrm{L}$ pipette to generate a gap, washed with PBS, and supplied with growth medium. Before and after $22 \mathrm{~h}$ of culture, images of the scratched area were taken using a microscope (Leica Microsystems, Wetzlar, Germany). The filled area was measured using ImageJ software.

\subsection{Matrigel Invasion Assay}

In vitro invasion potential was analyzed using a Transwell chamber culture system with $8-\mu \mathrm{m}$ pores for a 24-well plate (BD BioCoat ${ }^{\mathrm{TM}}$ Matrigel $^{\mathrm{TM}}$ Invasion Chamber; Becton, Dickinson \& Company, Franklin Lakes, NJ, USA), as described previously [10]. The upper chambers were placed on culture plates with 24 wells filled with $0.75 \mathrm{~mL}$ of the conditioned medium of G3H cells [10], and cells $\left(2 \times 10^{4}\right.$ cells in the control chamber; $1 \times 10^{5}$ cells in the Matrigel chamber) suspended in $0.5 \mathrm{~mL}$ of serum-free DMEM were added to the upper chamber. After $22 \mathrm{~h}$ of culture, the migrated cells on the lower surface of the filter of the upper chambers were fixed and counted using a fluorescence microscope (Leica Microsystems) at $\times 100$ magnification, and the average number of cells in four fields was taken for each well.

\subsection{Western Blotting}

Western blotting was performed as described previously [11]. Protein extracts were separated by SDS-PAGE and transferred to polyvinylidene difluoride membranes (Merck KGaA, Darmstadt, Germany). The membranes were immunoblotted with a rabbit polyclonal antibody specific for IFITM1 (ab106265, Abcam, Cambridge, UK) and a mouse monoclonal antibody specific for $\alpha$-tubulin (T5168, Sigma-Aldrich, St. Louis, MO, USA). 


\subsection{Immunohistochemistry}

A human SCLC tissue microarray (BS04116) was purchased from US Biomax (Derwood, MD, USA). Immunohistochemical staining was performed as previously described [10,11]. Briefly, the slides were deparaffinized and rehydrated, followed by antigen unmasking with citrate buffer. The arrays were blocked with $10 \%$ horse serum and incubated with a primary antibody against IFITM1 (ab106265, Abcam) overnight at $4^{\circ} \mathrm{C}$. Anti-rabbit Ig from the ImmPRESS Reagent Kit and ImmPACT DAB (Vector Laboratories) were used for antigen-antibody detection, and then the slides were briefly immersed in hematoxylin for counterstaining and evaluated under a light microscope.

\subsection{Isolation of Human Tumor Cells from Tumor Tissues}

For the isolation of human tumor cells from tumor tissues of the orthotropic metastasis model mice, we used the MACS ${ }^{\circledR}$ cell separation technology (Miltenyi Biotec, Bergish Gladbach, Germany) according to the manufacturer's instructions. Briefly, orthotropic and metastatic tumor tissues of the mice were resected and minced into small pieces under sterile conditions. The tumor pieces were cultured 4-10 days in DMEM containing 10\% FBS, and the cells were trypsinized and suspended in PBS containing 0.5\% bovine serum albumin and $2 \mathrm{mM}$ EDTA. Anti-mouse MHC class I H-2 Dd PE-conjugated antibody (Acris antibodies, Herford, Germany) was added to the cell suspension to label mouse cells, and the labeled cells were captured by anti-PE Microbeads (Miltenyi Biotec). The suspension was applied to the MACS ${ }^{\circledR}$ LD column (Miltenyi Biotec), and the flow-through containing the unlabeled human tumor cells was collected.

\subsection{RNA Isolation and Real-Time RT-PCR}

Total RNA was isolated from cells using an RNeasy Plus Kit (Qiagen, Hilden, Germany). cDNA was synthesized from total RNA using a Reverse Transcription System (Promega, Madison, WI, USA). Real-time RT-PCR was performed using TB Green ${ }^{\circledR}$ Premix Ex Taq ${ }^{\mathrm{TM}}$ II (Takara Bio, Shiga, Japan) and a Thermal Cycler Dice Real Time System (Takara Bio). All primers were purchased from Takara Bio. All reactions were run in at least duplicate, and the relative expression levels were calculated by the $\Delta \triangle C T$ method using beta-actin as a reference.

\subsection{DNA Microarray Analysis}

DNA microarray analysis was performed as described previously [44] with some modifications. Briefly, double-stranded cDNA was synthesized using a Low Input Quick Amp Labeling Kit, One-color (Agilent Technologies, Santa Clara, CA, USA) from 200 ng of total RNA with oligo (dT) primer and amplified with T7 RNA polymerase to generate up to approximately $10 \mu \mathrm{g}$ of cRNA. The labeled cRNAs were hybridized to the SurePrint G3 Human GE $8 \times 60 \mathrm{~K}$ oligonucleotide microarray (Agilent Technologies) according to the manufacturer's instructions. The raw microarray data were analyzed using GeneSpring GX software (Agilent Technologies).

\subsection{Lentivirus Preparation}

To prepare VSV-G pseudotyped replication-defective lentiviruses, HEK293T cells were co-transfected with lentiviral vectors, Gag/pol, and Env plasmids using FuGeneHD (Promega). For shRNA expression, we constructed SV40 promoter-driven puromycin-expressing lentivirus vector based from pLenti6/V5-GW/lacZ (Invitrogen). An shRNA expression cassette was inserted into the defective U3 region of the $3^{\prime}$ long terminal repeat of the vector, and shRNAs were transcribed from the mouse U6 promoter. The target sequences of shRNAs were as follows: shIFITM1, 5'-CCTAGATACAGCAGTTTATAC-3'; and shLacZ, 5'-GCAGTTATCTGGAAGATCAGG-3'. For cDNA expression, the CSII-CMV-MCS-IRES2-Bsd vector, kindly gifted by H. Miyoshi (RIKEN Tsukuba Institute), was used. IFITM1 cDNA was amplified from a cDNA pool of DMS273 cells by PCR and cloned into CSII-CMV-MCS-IRES2-Bsd at the NheI and XhoI sites. 


\subsection{Animal Experiments}

Animal experiments were approved by the Institute Committee for Animal Experiments at the Institute of Microbial Chemistry and conducted according to the ethics guidelines of our institute. Female BALB/c nude mice were obtained from Charles River Japan (Kanagawa, Japan). Mice aged 8 weeks were used for the in vivo anti-tumor assay. The orthotopic and subcutaneous xenograft models were prepared as described previously $[10,11]$. To generate the experimental metastasis model, $1 \times 10^{6}$ DMS273-GFP cells or $4 \times 10^{6} \mathrm{H69ZN}$ cells in $200 \mu \mathrm{L}$ of serum-free medium were injected into the tail veins of nude mice. An Olympus OV110 Small Animal Imaging System (Olympus, Tokyo, Japan) was used to image tumor formation in the mice. The length (L) and width (W) of the orthotopic and subcutaneous tumors were measured using calipers, and the tumor volume (TV) was calculated using the following formula: $\mathrm{TV}=\left(\mathrm{L} \times \mathrm{W}^{2}\right) / 2$. The incidence of distant metastasis was calculated as the number of distant metastasis-positive mice divided by number of total mice in the experimental group.

\subsection{Statistical Analysis}

Statistical analysis was conducted using Fisher's exact test or the Mann-Whitney U-test. $p<0.05$ denoted statistical significance.

Supplementary Materials: Supplementary materials can be found at http://www.mdpi.com/1422-0067/21/14/ 4934/s1. Supplemental Figure S1; IFITM1 overexpression on the metastatic phenotype of DMS273-GFP cells. Supplemental Figure S2; Effect of IFITM1 silencing on the metastatic phenotype of DMS273-GFP cells.

Author Contributions: Conceptualization, S.S. and M.K.; methodology, S.S. and T.M.; investigation, S.S., H.I., Y.K., and S.-i.O.; writing—original draft preparation, S.S.; writing—review and editing, M.K.; funding acquisition, S.S. All authors have read and agreed to the published version of the manuscript.

Funding: This work was supported by JSPS KAKENHI Grant Number 26460481 and 17K08777.

Acknowledgments: We thank H. Miyoshi (RIKEN) for providing the CSII-CMV-MCS-IRES2-Bsd vector. We also thank Y. Hayakawa (Toyama Univ.) and S. Takagi (JFCR) for helpful discussions. We also thank I. Momose, T. Ohishi, Ms. I. Usami and Ms. A. Harakawa (BIKAKEN) for technical support. We thank Joe Barber Jr., from Edanz Group (https://en-author-services.edanzgroup.com/) for editing a draft of this manuscript.

Conflicts of Interest: The authors declare no conflict of interest.

\section{Abbreviations}

$\begin{array}{ll}\text { CTA } & \text { cancer tissue array } \\ \text { IFITM1 } & \text { interferon-induced transmembrane protein 1 } \\ \text { IFN } & \text { interferon } \\ \text { NSCLC } & \text { non-small cell lung cancer } \\ \text { SCLC } & \text { small cell lung cancer }\end{array}$

\section{References}

1. Byers, L.A.; Rudin, C.M. Small cell lung cancer: Where do we go from here? Cancer 2015, 121, 664-672. [CrossRef] [PubMed]

2. Gazdar, A.F.; Bunn, P.A.; Minna, J.D. Small-cell lung cancer: What we know, what we need to know and the path forward. Nat. Rev. Cancer 2017, 17, 725-737. [CrossRef] [PubMed]

3. Sabari, J.K.; Lok, B.H.; Laird, J.H.; Poirier, J.T.; Rudin, C.M. Unravelling the biology of SCLC: Implications for therapy. Nat. Rev. Clin. Oncol. 2017, 14, 549-561. [CrossRef] [PubMed]

4. Verma, V.; Sharma, G.; Singh, A. Immunotherapy in extensive small cell lung cancer. Exp. Hematol. Oncol. 2019, 8, 5. [CrossRef] [PubMed]

5. Kuramoto, T.; Goto, H.; Mitsuhashi, A.; Tabata, S.; Ogawa, H.; Uehara, H.; Saijo, A.; Kakiuchi, S.; Maekawa, Y.; Yasutomo, K.; et al. Dll4-Fc, an inhibitor of Dll4-notch signaling, suppresses liver metastasis of small cell lung cancer cells through the downregulation of the NF-kappaB activity. Mol. Cancer Ther. 2012, 11, 2578-2587. [CrossRef] [PubMed] 
6. Li, B.; Wang, C.; Zhang, Y.; Zhao, X.Y.; Huang, B.; Wu, P.F.; Li, Q.; Li, H.; Liu, Y.S.; Cao, L.Y.; et al. Elevated PLGF contributes to small-cell lung cancer brain metastasis. Oncogene 2013, 32, 2952-2962. [CrossRef] [PubMed]

7. Kwon, M.C.; Proost, N.; Song, J.Y.; Sutherland, K.D.; Zevenhoven, J.; Berns, A. Paracrine signaling between tumor subclones of mouse SCLC: A critical role of ETS transcription factor Pea3 in facilitating metastasis. Genes Dev. 2015, 29, 1587-1592. [CrossRef] [PubMed]

8. Denny, S.K.; Yang, D.; Chuang, C.H.; Brady, J.J.; Lim, J.S.; Gruner, B.M.; Chiou, S.H.; Schep, A.N.; Baral, J.; Hamard, C.; et al. Nfib Promotes Metastasis through a Widespread Increase in Chromatin Accessibility. Cell 2016, 166, 328-342. [CrossRef]

9. Semenova, E.A.; Kwon, M.C.; Monkhorst, K.; Song, J.Y.; Bhaskaran, R.; Krijgsman, O.; Kuilman, T.; Peters, D.; Buikhuisen, W.A.; Smit, E.F.; et al. Transcription Factor NFIB Is a Driver of Small Cell Lung Cancer Progression in Mice and Marks Metastatic Disease in Patients. Cell Rep. 2016, 16, 631-643. [CrossRef]

10. Sakamoto, S.; Inoue, H.; Ohba, S.; Kohda, Y.; Usami, I.; Masuda, T.; Kawada, M.; Nomoto, A. New metastatic model of human small-cell lung cancer by orthotopic transplantation in mice. Cancer Sci. 2015, 106, 367-374. [CrossRef]

11. Sakamoto, S.; Inoue, H.; Kaneko, M.K.; Ogasawara, S.; Kajikawa, M.; Urano, S.; Ohba, S.I.; Kato, Y.; Kawada, M. Generation and evaluation of a chimeric antibody against coxsackievirus and adenovirus receptor for cancer therapy. Cancer Sci. 2019, 110, 3595-3602. [CrossRef] [PubMed]

12. Carney, D.N.; Gazdar, A.F.; Bepler, G.; Guccion, J.G.; Marangos, P.J.; Moody, T.W.; Zweig, M.H.; Minna, J.D. Establishment and identification of small cell lung cancer cell lines having classic and variant features. Cancer Res. 1985, 45, 2913-2923.

13. Pedersen, N.; Mortensen, S.; Sørensen, S.B.; Pedersen, M.W.; Rieneck, K.; Bovin, L.F.; Poulsen, H.S. Transcriptional gene expression profiling of small cell lung cancer cells. Cancer Res. 2003, 63, 1943-1953. [PubMed]

14. Shtivelman, E.; Namikawa, R. Species-specific metastasis of human tumor cells in the severe combined immunodeficiency mouse engrafted with human tissue. Proc. Natl. Acad. Sci. USA 1995, 92, 4661-4665. [CrossRef]

15. Deblandre, G.A.; Marinx, O.P.; Evans, S.S.; Majjaj, S.; Leo, O.; Caput, D.; Huez, G.A.; Wathelet, M.G. Expression cloning of an interferon-inducible 17-kDa membrane protein implicated in the control of cell growth. J. Biol. Chem. 1995, 270, 23860-23866. [CrossRef] [PubMed]

16. Diamond, M.S.; Farzan, M. The broad-spectrum antiviral functions of IFIT and IFITM proteins. Nat. Rev. Immunol. 2013, 13, 46-57. [CrossRef]

17. Kampen, K.R. Membrane proteins: The key players of a cancer cell. J. Membr. Biol. 2011, 242, 69-74. [CrossRef]

18. Brass, A.L.; Huang, I.C.; Benita, Y.; John, S.P.; Krishnan, M.N.; Feeley, E.M.; Ryan, B.J.; Weyer, J.L.; van der Weyden, L.; Fikrig, E.; et al. The IFITM proteins mediate cellular resistance to influenza A H1N1 virus, West Nile virus, and dengue virus. Cell 2009, 139, 1243-1254. [CrossRef]

19. Lewin, A.R.; Reid, L.E.; McMahon, M.; Stark, G.R.; Kerr, I.M. Molecular analysis of a human interferon-inducible gene family. Eur. J. Biochem. 1991, 199, 417-423. [CrossRef]

20. Huang, I.C.; Bailey, C.C.; Weyer, J.L.; Radoshitzky, S.R.; Becker, M.M.; Chiang, J.J.; Brass, A.L.; Ahmed, A.A.; Chi, X.; Dong, L.; et al. Distinct patterns of IFITM-mediated restriction of filoviruses, SARS coronavirus, and influenza A virus. PLoS Pathog. 2011, 7, e1001258. [CrossRef]

21. Tanaka, S.S.; Yamaguchi, Y.L.; Tsoi, B.; Lickert, H.; Tam, P.P. IFITM/Mil/fragilis family proteins IFITM1 and IFITM3 play distinct roles in mouse primordial germ cell homing and repulsion. Dev. Cell. 2005, 9, 745-756. [CrossRef]

22. Hatano, H.; Kudo, Y.; Ogawa, I.; Tsunematsu, T.; Kikuchi, A.; Abiko, Y.; Takata, T. IFN-induced transmembrane protein 1 promotes invasion at early stage of head and neck cancer progression. Clin. Cancer Res. 2008, 14, 6097-6105. [CrossRef]

23. Yu, F.; Xie, D.; Ng, S.S.; Lum, C.T.; Cai, M.Y.; Cheung, W.K.; Kung, H.F.; Lin, G.; Wang, X.; Lin, M.C. IFITM1 promotes the metastasis of human colorectal cancer via CAV-1. Cancer Lett. 2015, 368, 135-143. [CrossRef]

24. Sari, I.N.; Yang, Y.G.; Phi, L.T.H.; Kim, H.; Baek, M.J.; Jeong, D.; Kwon, Y.H. Interferon-induced transmembrane protein 1 (IFITM1) is required for the progression of colorectal cancer. Oncotarget 2016, 7, 86039-86050. [CrossRef]

25. Yang, Y.G.; Koh, Y.W.; Sari, I.N.; Jun, N.; Lee, S.; Phi, L.T.H.; Kim, K.S.; Wijaya, Y.T.; Lee, S.H.; Baek, M.J.; et al. Interferon-induced transmembrane protein 1-mediated EGFR/SOX2 signaling axis is essential for progression of non-small cell lung cancer. Int. J. Cancer 2019, 144, 2020-2032. [CrossRef] 
26. Yan, J.; Jiang, Y.; Lu, J.; Wu, J.; Zhang, M. Inhibiting of Proliferation, Migration, and Invasion in Lung Cancer Induced by Silencing Interferon-Induced Transmembrane Protein 1 (IFITM1). Biomed. Res. Int. 2019, 2019, 9085435. [CrossRef]

27. Li, H.; Yu, B.; Li, J.; Su, L.; Yan, M.; Zhu, Z.; Liu, B. Overexpression of IncRNA H19 enhances carcinogenesis and metastasis of gastric cancer. Oncotarget 2014, 5, 2318-2329. [CrossRef]

28. Raveh, E.; Matouk, I.J.; Gilon, M.; Hochberg, A. The H19 Long non-coding RNA in cancer initiation, progression and metastasis - a proposed unifying theory. Mol. Cancer 2015, 14, 184. [CrossRef]

29. Zhang, H.; Yu, Y.; Zhang, K.; Liu, X.; Dai, Y.; Jiao, X. Targeted inhibition of long non-coding RNA H19 blocks anaplastic thyroid carcinoma growth and metastasis. Bioengineered. 2019, 10, 306-315. [CrossRef]

30. Li, X.; Lv, F.; Li, F.; Du, M.; Liang, Y.; Ju, S.; Liu, Z.; Wang, B.; Gao, Y. Long Noncoding RNA H19 Facilitates Small Cell Lung Cancer Tumorigenesis Through miR-140-5p/FGF9 Axis. Onco. Targets Ther. 2020, 13, 3525-3534. [CrossRef]

31. Ranjbar, S.; Haridas, V.; Jasenosky, L.D.; Falvo, J.V.; Goldfeld, A.E. A Role for IFITM Proteins in Restriction of Mycobacterium tuberculosis Infection. Cell Rep. 2015, 13, 874-883. [CrossRef] [PubMed]

32. Yang, G.; Xu, Y.; Chen, X.; Hu, G. IFITM1 plays an essential role in the antiproliferative action of interferon-gamma. Oncogene 2007, 26, 594-603. [CrossRef] [PubMed]

33. Zhao, X.; Li, J.; Winkler, C.A.; An, P.; Guo, J.T. IFITM genes, variants, and their roles in the control and pathogenesis of viral infections. Front. Microbiol. 2019, 9, 3228. [CrossRef] [PubMed]

34. Li, D.; Peng, Z.; Tang, H.; Wei, P.; Kong, X.; Yan, D.; Huang, F.; Li, Q.; Le, X.; Li, Q.; et al. KLF4-mediated negative regulation of IFITM3 expression plays a critical role in colon cancer pathogenesis. Clin. Cancer Res. 2011, 17, 3558-3568. [CrossRef] [PubMed]

35. Min, J.; Feng, Q.; Liao, W.; Liang, Y.; Gong, C.; Li, E.; He, W.; Yuan, R.; Wu, L. IFITM3 promotes hepatocellular carcinoma invasion and metastasis by regulating MMP9 through p38/MAPK signaling. FEBS Open Bio. 2018, 8, 1299-1311. [CrossRef] [PubMed]

36. Liu, X.; Chen, L.; Fan, Y.; Hong, Y.; Yang, X.; Li, Y.; Lu, J.; Lv, J.; Pan, X.; Qu, F.; et al. IFITM3 promotes bone metastasis of prostate cancer cells by mediating activation of the TGF- $\beta$ signaling pathway. Cell Death Dis. 2019, 10, 517. [CrossRef]

37. Yanez, D.C.; Ross, S.; Crompton, T. The IFITM protein family in adaptive immunity. Immunology 2020, 159, 365-372. [CrossRef]

38. Yu, F.; Ng, S.S.; Chow, B.K.; Sze, J.; Lu, G.; Poon, W.S.; Kung, H.F.; Lin, M.C. Knockdown of interferon-induced transmembrane protein 1 (IFITM1) inhibits proliferation, migration, and invasion of glioma cells. J. Neurooncol. 2011, 103, 187-195. [CrossRef]

39. Kim, N.H.; Sung, H.Y.; Choi, E.N.; Lyu, D.; Choi, H.J.; Ju, W.; Ahn, J.H. Aberrant DNA methylation in the IFITM1 promoter enhances the metastatic phenotype in an intraperitoneal xenograft model of human ovarian cancer. Oncol. Rep. 2014, 31, 2139-2146. [CrossRef]

40. Frey, M.A.; Appenheimer, M.M.; Evans, S.S. Tyrosine kinase-dependent regulation of L-selectin expression through the Leu-13 signal transduction molecule: Evidence for a protein kinase C-independent mechanism of L-selectin shedding. J. Immunol. 1997, 158, 5424-5434.

41. Wilkins, C.; Woodward, J.; Lau, D.T.; Barnes, A.; Joyce, M.; McFarlane, N.; McKeating, J.A.; Tyrrell, D.L.; Gale, M., Jr. IFITM1 is a tight junction protein that inhibits hepatitis C virus entry. Hepatology 2013, 57, 461-469. [CrossRef] [PubMed]

42. Yang, Y.; Lee, J.H.; Kim, K.Y.; Song, H.K.; Kim, J.K.; Yoon, S.R.; Cho, D.; Song, K.S.; Lee, Y.H.; Choi, I. The interferon-inducible 9-27 gene modulates the susceptibility to natural killer cells and the invasiveness of gastric cancer cells. Cancer Lett. 2005, 221, 191-200. [CrossRef] [PubMed]

43. Cheon, H.; Borden, E.C.; Stark, G.R. Interferons and their stimulated genes in the tumor microenvironment. Semin. Oncol. 2014, 41, 156-173. [CrossRef] [PubMed]

44. Sakamoto, S.; Kojima, F.; Momose, I.; Kawada, M.; Adachi, H.; Nishimura, Y. Decalpenic acid induces early osteoblastic markers in pluripotent mesenchymal cells via activation of retinoic acid receptor gamma. Biochem. Biophys. Res. Commun. 2012, 422, 751-757. [CrossRef] [PubMed]

(C) 2020 by the authors. Licensee MDPI, Basel, Switzerland. This article is an open access article distributed under the terms and conditions of the Creative Commons Attribution (CC BY) license (http://creativecommons.org/licenses/by/4.0/). 